\title{
Performance predictions for electromagnetic launching with multi-fibre solid brush armatures and resistively layered rail accelerators
}

\author{
A.J. Schoolderman \\ TNO PML-Pulse Physics, Delft, the Netherlands
}

\begin{abstract}
In the literature on EML research, a number of proposals have been made to suppress the negative influence of the velocity skin effect on the performance of solid armatures during electromagnetic launch. In this paper, the results of a study of two of these methods, i.e. the application of multi-fibre solid brush armatures and the use of accelerator rails with a resistive layer, are presented. This study is performed by means of two-dimensional finite element computer simulations of the electrothermal behaviour of the armature and the rails during the launch process. A description of the electrothermal model used in the simulations is given. Here, the fibre armatures are regarded as made of materials with an anisotropic electrical and thermal conductivity. The results for the current distribution in a rectangular multi-fibre solid brush armature obtained from the simulations agree with the results of an analytical method. It is shown that fibre armatures have a more homogeneous current distribution during the acceleration process than monobloc armatures. $U$. shaped molybdenum multi-fibre solid brush armatures are good candidates for arc erosion-free launching if the electrical insulation of the fibres can be maintained at increasing temperature. Simulations also show that the skin depth in monobloc and fibre armatures can be increased by using rails with a resistive layer with suitable material properties.
\end{abstract}

\section{INTRODUCTION}

One of the key challenges in electromagnetic launch research with solid armatures today is to achieve acceleration up to velocities of $2.5 \mathrm{~km} / \mathrm{s}$ and higher without erosion caused by arcing. This ambitious goal can only be reached when the transition of the solid armature into a hybrid one takes place in a controllable way or when the transition is of avoided entirely. A widely accepted opinion is that this transition is triggered by (partial) melting of the rail-armature interface caused by the high current density at the trailing edge of the armature. This locally high current density results from the velocity skin effect. In order to suppress this negative influence the velocity skin effect on the performance of solid armatures during electromagnetic launching, a number of proposals with respect to the choice of the armature geometry and the armature-rail material combination are made in the literature.

In this paper we focus on two of these proposals: the multi-fibre solid brush armature and rails with a resistive layer. The effect on the electrothermal behaviour of the

Manuscript received February 16, 1994.

A.J. Schoolderman, TNO-PML Pulse Physics, mailing address: P.O. Box 45, 2280 AA Rijswijk, the Netherlands, vistiting address: Schoemakerstraat 97 2628 VK Delft, the Netherlands, phone: +31 15697011 , fax: +31 15 621268 . armature during launch when fibre armatures and a resistive layer on the rails are applied, is studied by means of 2dimensional computer simulations. The model on which the simulations are based, is described in section II. Results of the simulations of fibre armatures are presented in sections III and IV. In section V, electromagnetic launching with a resistive layer on the rails is discussed.

\section{2-D MODEL}

In this section, we give a description of the twodimensional model on which the finite element computer code used for the simulations, is based. The computer code was originally developed by G.C. Long [1], [2]. In the model, the $x-y$ plane at half-height of the dipole rail accelerator is considered. Here, the $x$-direction is chosen along the accelerator and the $y$-direction is perpendicular to the railarmature interface (see Fig. 1). In this plane, the only component of the magnetic field $\boldsymbol{H}$ unequal to zero, is the $z-$ component $H_{\mathrm{z}}(x, y, t)$.

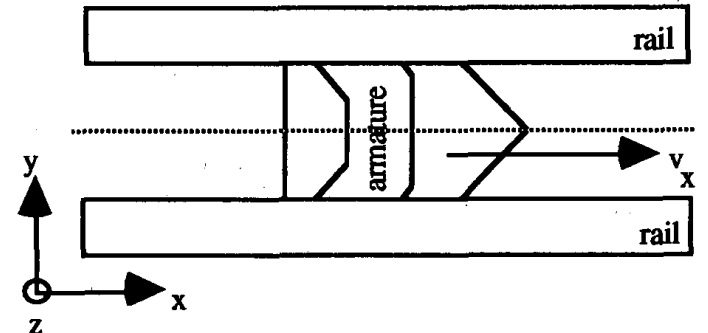

Fig.1. Outline of the two-dimensional accelerator and ammature geometry.

From Maxwell's equations, where the displacement current term is omitted, and Ohm's law,

$$
J=\underline{\rho}^{-1} \cdot(E+v \times B),
$$

a partial differential equation for $H_{\mathrm{z}}$ can be derived. Here, $J$, $\boldsymbol{E}, \boldsymbol{v}$ and $\boldsymbol{B}$ are the current density, the electric field, the armature velocity and the magnetic induction, respectively. The resistivity tensor is denoted by $\rho$. The equation for $H_{\mathrm{z}}$ reads

$$
\begin{array}{r}
\left(\rho_{\mathrm{yy}} \frac{\partial^{2}}{\partial x^{2}}+\rho_{\mathrm{xx}} \frac{\partial^{2}}{\partial y^{2}}-\left(\rho_{\mathrm{xy}}+\rho_{\mathrm{yx}}\right) \frac{\partial^{2}}{\partial x \partial y}\right) H_{\mathrm{z}} \\
-\mu_{0} v_{\mathrm{x}} \frac{\partial}{\partial x} H_{\mathrm{z}}=\mu_{0} \frac{\partial}{\partial t} H_{\mathrm{z}},
\end{array}
$$

where we used $v(t)=\left(v_{\mathrm{X}}(t), 0,0\right)$. The $i$-j-components of the resistivity tensor are denoted by $\rho_{i j}$ and $\mu_{0}$ is the magnetic permeability of vacuum. For materials with an isotropic resistivity, i.e. $\rho=\rho \underline{U}$ with $\rho$ the scalar resistivity and $\underline{U}$ the unit tensor, eq. (2) reduces to 


$$
\rho\left(\frac{\partial^{2}}{\partial x^{2}}+\frac{\partial^{2}}{\partial y^{2}}\right) H_{z}-\mu_{0} v_{x} \frac{\partial}{\partial x} H_{z}=\mu_{0} \frac{\partial}{\partial t} H_{z}
$$

A partial differential equation with a strong mathematical resemblance to eq. (2) can be derived for the temperature distribution in the $x$-y-plane, $T(x, y, t)$, from the energy balance equation and Fourier's law:

$$
J_{\mathrm{q}}=-\underline{\kappa} \cdot \nabla T \text {. }
$$

Here, $J_{\mathrm{q}}$ is the energy flow and $\underline{\kappa}$ is the thermal conductivity tensor. The equation for $T$ reads

$$
\begin{aligned}
\left(\kappa_{\mathrm{xx}} \frac{\partial^{2}}{\partial x^{2}}+\kappa_{\mathrm{yy}} \frac{\partial^{2}}{\partial y^{2}}+\left(\kappa_{\mathrm{xy}}+\kappa_{\mathrm{yx}}\right) \frac{\partial^{2}}{\partial x \partial y}\right) T \\
-c_{\mathrm{v}} \nu_{\mathrm{x}} \frac{\partial}{\partial x} T=c_{\mathrm{v}} \frac{\partial}{\partial t} T-\mathrm{f}_{\text {source }},
\end{aligned}
$$

with $c_{v}$ the specific heat per unit volume. In the case of Joule heating, the source term can be written as

$$
\mathrm{f}_{\text {source }}=\rho_{\mathrm{xx}} J_{\mathrm{x}}^{2}+\rho_{\mathrm{yy}} J_{\mathrm{y}}^{2}+\left(\rho_{\mathrm{xy}}+\rho_{\mathrm{yx}}\right) J_{\mathrm{x}} J_{\mathrm{y}} \text {. }
$$

Heat sources other than Joule heating are not taken into account here. For materials with an isotropic thermal conductivity, eq. (5) reduces to

$$
\kappa\left(\frac{\partial^{2}}{\partial x^{2}}+\frac{\partial^{2}}{\partial y^{2}}\right) T-c_{\mathrm{v}} v_{\mathrm{x}} \frac{\partial}{\partial x} T=c_{\mathrm{v}} \frac{\partial}{\partial t} T-\rho J^{2},
$$

with $J=|J|$. The diffusion-type equations for the magnetic field (2) and for the temperature distribution (5) are coupled by the source term (6) and by the temperature and spatial dependence of the material properties $\rho_{\mathrm{ij}}, \kappa_{\mathrm{ij}}$ and $c_{\mathrm{v}}$. With the appropriate initial and boundary conditions, these equations can be solved with the computer code simultaneously. For the simulations presented in this paper, the velocity $v_{\mathrm{X}}(t)$ is given as input data.

To simulate the electrothermal behaviour of multi-fibre solid brush armatures, the following model for this type of armatures is used.

- The electrical conductivity in the direction of the fibres, $\sigma_{/ /}$, is very large compared to the electrical conductivity perpendicular to the fibres, $\sigma_{\perp}$. The ratio of these conductivities $R=\sigma_{/ /} / \sigma_{\perp}$ is chosen equal to $10^{3}$.

- The ratio of the thermal conductivity in the direction of the fibres, $\kappa_{/ /}$, and the thermal conductivity perpendicular to the fibres, $\kappa_{\perp}$, is also chosen equal to $10^{3}$.

- The mass density ratio $r$ is defined as the ratio of the mass density of a fibre armature to the mass density of a monobloc armature made of the same material. The electrical conductivity in the direction of the fibres, $\sigma_{/ /}$, and the specific heat per volume, $c_{\mathrm{V}}$, of a fibre armature are taken equal to $r$ times the values of the electrical conductivity and the specific heat of a monobloc armature made of the same material and with the same volume:

$$
\begin{aligned}
& \sigma_{/ I}^{\text {fibre }}=r \cdot \sigma^{\text {monobloc }}, \\
& c_{\mathrm{v}}^{\text {fibre }}=r \cdot c_{\mathbf{v}}^{\text {monobloc }} .
\end{aligned}
$$

In the following sections, results of simulations of electromagnetic launching with multi-fibre solid brush armatures based on this model are presented.

\section{COMPARISON BETWEEN THE FINITE ELEMENT METHOD AND THE ANALYTICAL METHOD OF HUGHES AND YOUNG}

In 1982, a paper by W.F. Hughes and F.J. Young was published in which a method was presented to calculate the magnetic field distribution in the two-dimensional representation of the electromagnetic launch process (see Fig. 1) with rectangular multi-fibre solid brush armatures [3]. Here, the fibre armatures are considered to be 'infinitely laminated', i.e. composed of an infinite number of parallel, insulated fibres, and the electrical conductivity in the direction perpendicular to the fibres, $\sigma_{\perp}=\sigma_{x x}$, is taken equal to zero. The method of Hughes and Young can only solve the diffusion problem for the magnetic field in rectangular fibre armatures. Moreover, no convergence criterion has been established for the governing integro-differential equation. Hence, we are limited to a comparison of results of our finite element simulations to the results published in [3]: the magnetic field $H_{\mathrm{z}}(x, t)$ in a copper, rectangular fibre armature with a rail-armature interface length $l$ of $0.01 \mathrm{~m}$. The electrical conductivity tensor for the armature material has only one component unequal to zero, i.e. $\sigma_{/ /}=\sigma_{\mathrm{yy}}=$ $5 \cdot 10^{7} /(\Omega \mathrm{m})$. The rail accelerator has copper rails $(\sigma=$ $5 \cdot 10^{7} /(\Omega \mathrm{m})$ ) and the inter-rail distance is $0.04 \mathrm{~m}$. The conductivity for both the rails and the armature is assumed to be uniform and independent of the temperature. The breech current, which mimics a discharge of a capacitor bank, decreases linearly from its initial value $I_{0}$ to $70 \%$ of that value in $3 \mathrm{~ms}$. In the same interval, the armature velocity increases linearly from zero to $3 \mathrm{~km} / \mathrm{s}$.

Figures 2 and 3 show the dimensionless magnetic field $H^{*}$, calculated with the finite element computer code. $H^{*}$, defined by

$$
H^{*}\left(x^{*}, t^{*}\right)=H_{\mathrm{z}}(x, t) / H_{\mathrm{z}}(x=0, t=0),
$$

is a function of the dimensionless distance in the $x$-direction $x^{*}=x / l$ and the dimensionless time $t^{*}=t /\left(\mu_{0 \sigma / /} l^{2}\right)$. Here, $x$ is the distance along the rail-armature distance, measured from the trailing edge of the armature. Figure 2 shows a close resemblance to the figures 5 and 6 of [3], which give $H^{*}$ as calculated with Hughes and Young's method. For small $t^{*}$, the magnetic field distribution is dominated by diffusion. In this time interval, the diffusion depth increases (see Fig. 2 upper). For velocities larger than approximately $200 \mathrm{~m} / \mathrm{s}$, the velocity skin effect becomes important and the penetration of the field decreases (see Fig. 2 lower). When the influence of the rise of the temperature dependent conductivity is taken into account, the magnetic field distribution for $t^{*}>0.025$ changes dramatically [4]. At $t^{*}=0.4774$, the skin depth $d$, defined as the distance in the $x$-direction, measured from the trailing edge of the armature along the interface, at which the value of the magnetic field equals $e^{-1} H_{\mathrm{Z}}(x=0)$, is more than twice the skin depth calculated in the simulation in which no temperature effects are included (see Fig. 3). 

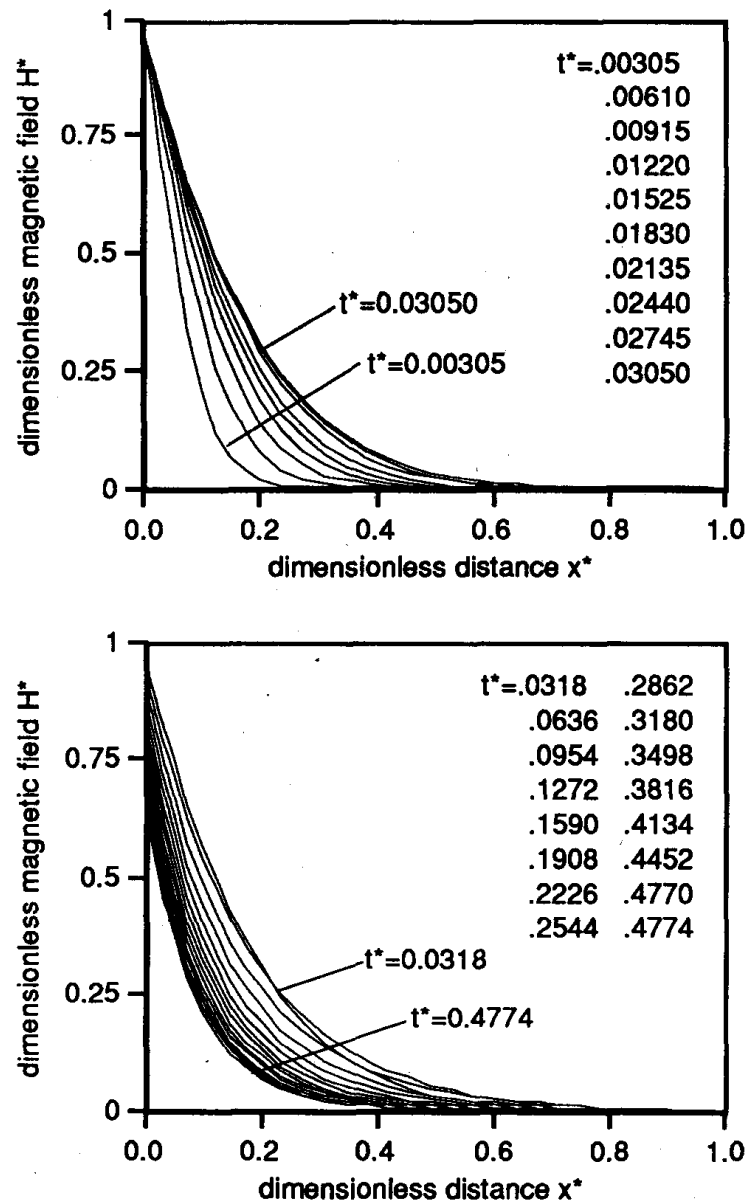

Fig. 2. Dimensionless magnetic field $H^{*}$ as a function of dimensionless distance $x^{*}$ for $0.00305 \leq t^{*} \leq 0.0305$ (upper) and $0.0318 \leq t^{*} \leq 0.4774$ (lower).

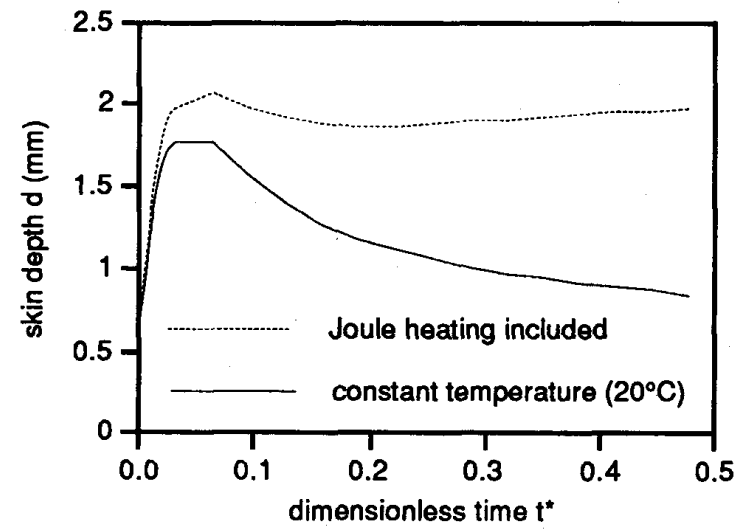

Fig. 3. Skin depth $d\left(t^{*}\right)$, calculated with the finite element method at constant temperature and by including the effect of the temperature rise, due to Joule heating, on the electrical conductivity.

\section{PERFORMANCE OF FIBRE ARMATURES}

In this section, results are presented of simulations of the electrothermal behaviour of multi-fibre solid brush armatures during electromagnetic launch with a $20 \mathrm{~mm}$ square-bore dipole rail accelerator with copper rails. A rectangular and 3 U-shaped armatures with trailing edge angles $\alpha$ (see Fig. 6a) of $30^{\circ}, 45^{\circ}$ and $60^{\circ}$ and made of copper, aluminium and molybdenum are considered. The mass of the complete launch package is chosen equal to $50 \mathrm{~g}$ in all simulations presented here. A breech current $I(t)$ is used which is typical for the current pulses used in the electromagnetic launch experiments at the Pulse Physics Laboratory. The breech current rises linearly from zero to approximately $35 \mathrm{kA}$ in $250 \mathrm{~ms}$. Then, a current pulse of $4 \mathrm{~ms}$ is generated with an amplitude of 275 kA. The armature starts to move when the breech current becomes equal to $100 \mathrm{kA}$, i.e. at $t=250.3 \mathrm{~ms}$ (Fig. 4).

The maximum temperature reached in a copper monobloc armature and a copper fibre armature during the simulated electromagnetic launch, is shown in Fig. 5. Both armatures have a trailing edge angle $\alpha$ of $45^{\circ}$ and an interface length of $15 \mathrm{~mm}$. Thermal insulation is not taken into account in these simulations, i.e. the thermal conductivity in the armatures is assumed to be isotropic. Results above the melting temperature of copper, denoted by the dashed line in the figure, are doubtful because of the limited modelling of the liquid state. Figures $6 \mathrm{a}, \mathrm{b}$ and $\mathrm{c}$ show snapshots of the temperature distribution in the fibre armature. The maximum temperature (e.g. $50^{\circ} \mathrm{C}$ in Fig. 6a) is denoted by black and the minimum temperature $\left(20^{\circ} \mathrm{C}\right)$ by white. The temperature distribution in a fibre armature is more uniform than in a monobloc armature (see [5] for snapshots of the temperature distribution in a monobloc armature). This phenomenon and the resulting lower maximum temperature in the fibre armature are caused by the more homogeneous current density in this armature. This is illustrated by Fig. 7, which shows the skin depth $d$ in the monobloc armature and the fibre armature during the launch.

In practice, the electrical insulation of the fibres will decrease when the local temperature exceeds, say, $200^{\circ} \mathrm{C}$. Fig. 5 also shows the maximum temperature in a $45^{\circ}$ copper fibre armature in which the resistivity perpendicular to the direction of the fibres, $\sigma_{\perp}$, decreases linearly between $150^{\circ} \mathrm{C}$ and $250^{\circ} \mathrm{C}$. At $250^{\circ} \mathrm{C}, \sigma_{\perp}$ is equal to 10 times the resistivity parallel to the fibre direction $\sigma_{\| / \text {. }}$.

From the results, we conclude that melting of the armature can be postponed by application of a fibre armature and that higher transition velocities can be achieved with fibre armatures than with monobloc armatures. Melting of the electrical insulation of the fibres during the acceleration process should be avoided by applying insulation materials which can withstand high temperatures.

In an earlier study, it was found that the smallest temperature rise in U-shaped monobloc armatures occurs in armatures with a large trailing edge angle $\alpha$. This is also valid for copper multi-fibre solid brush armatures: for the three armature geometries considered, the fibre armature with $\alpha=60^{\circ}$ is the last in which the melting temperature is reached (see Fig. 8). 


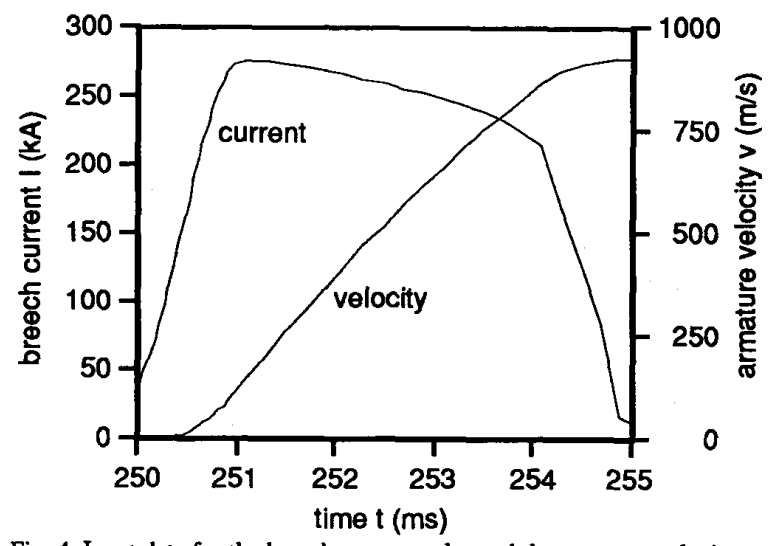

Fig. 4. Input data for the breech current pulse and the armature velocity.

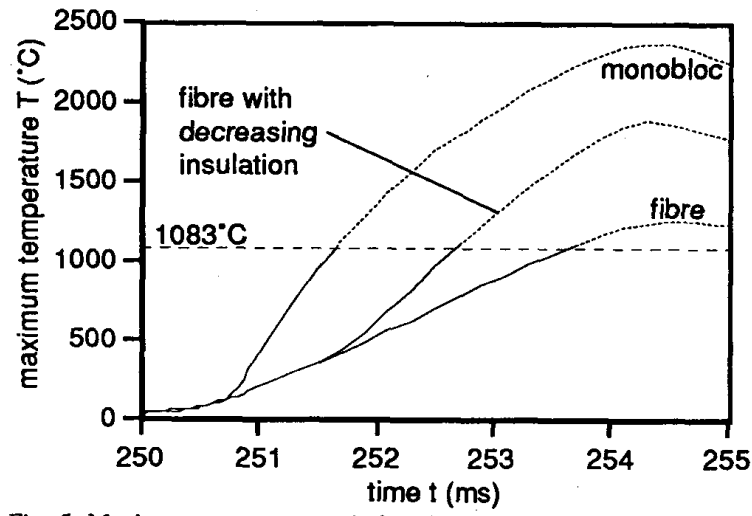

Fig. 5. Maximum temperatures during the simulated launch in three $45^{\circ}$ copper armatures with isotropic thermal conductivity.

By comparing the simulation results of electromagnetic launches with $45^{\circ}$ fibre armatures of different materials, the influence of the material choice on the electrothermal behaviour of the armatures is studied. The maximum temperatures in aluminium, copper and molybdenum fibre armatures during the launch process are depicted in Fig. 9. In the aluminium armature, melting starts at an early stage of the acceleration. The maximum temperature in the molybdenum armature is higher than in the other two armatures considered, but the high melting temperature of Mo of $2609^{\circ} \mathrm{C}$ is not reached.

In the simulations of which the results are presented here, it has been assumed that the fibres in the multi-fibre solid brush armatures are not thermally insulated. However, in practice the electrical insulation will also act as a thermal insulator. This will increase the maximum temperature in the armature, because the hot fibres can not give out their heat to their neighbouring fibres [4]. Therefore, heating of the fibres is nearly adiabatic.

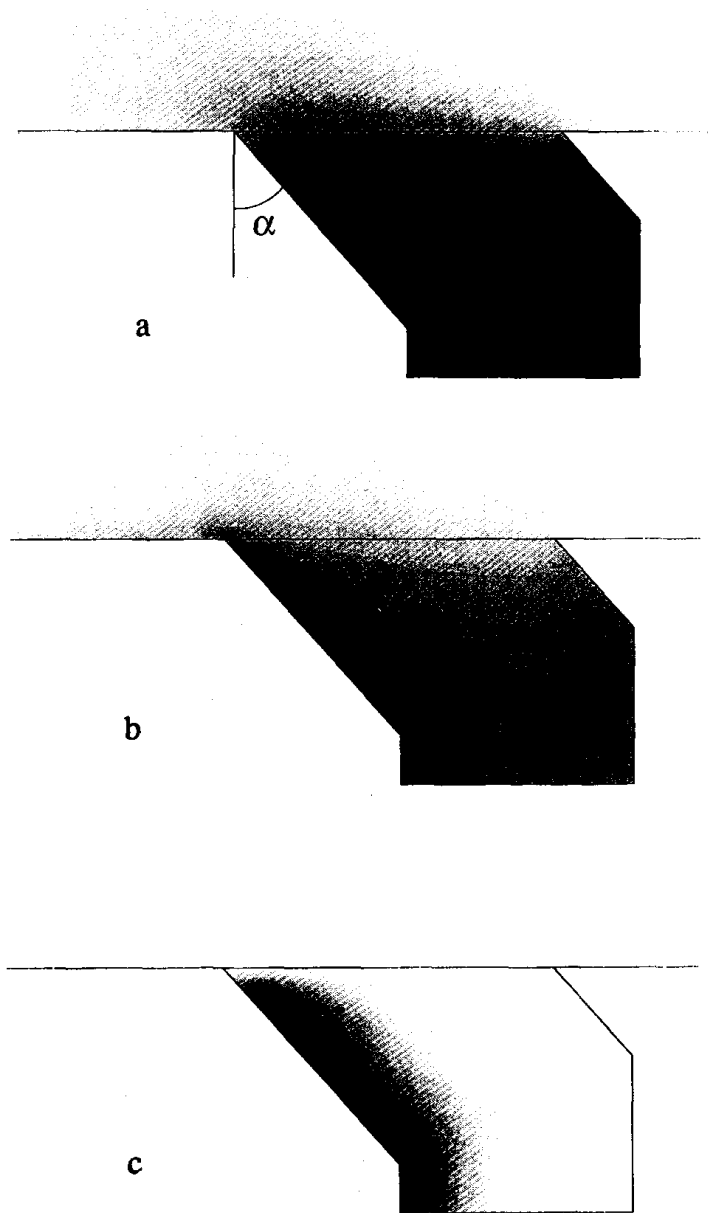

Fig. 6. Snapshots of the temperature distribution in a $45^{\circ}$, copper fibre armature at $t=250.00 \mathrm{~ms}$ (a), $250.28 \mathrm{~ms}$ (b), and $254.50 \mathrm{~ms}$ (c).

\section{PERFORMANCE OF RESISTIVELY LAYERED RAII ACCELERATORS}

It has been claimed by several authors that high transition velocities can be achieved when monobloc armatures in combination with rails with a resistive layer are applied in electromagnetic launching [6], [7], [8]. The resistive layer forces the current to penetrate deeper into the armature, so that the current density at its trailing edge is lower.

In contrast to this, it has been found in computer simulations that the performance of 'modified chevron' armatures, which consist of insulated leafs of e.g. copper or molybdenum with a thickness of approx. $1.0 \mathrm{~mm}$, with resistive rails (molybdenum layer on copper rails) is worse than with homogeneous copper rails, due to increased heating on the rail side of the rail-armature interface [1], [2]. However, no monobloc armatures or fibre armatures have been considered there.

In order to be able to compare results of our computer simulations with the predictions stated in [6], simulations of the magnetic field distribution in rectangular copper 


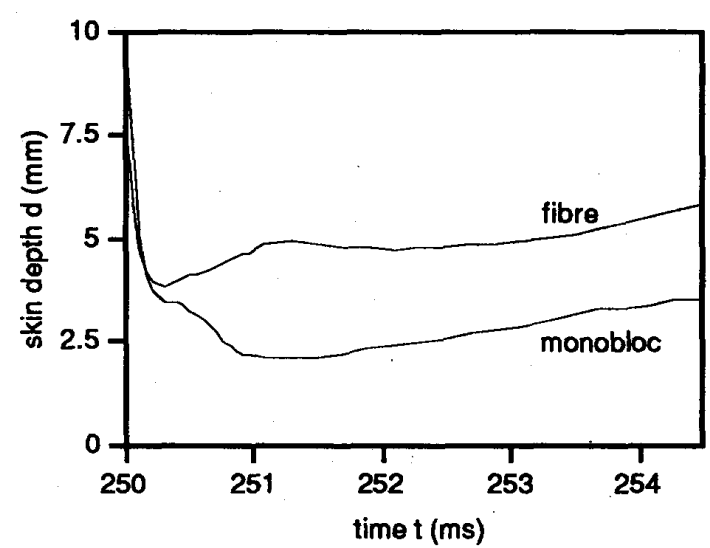

Fig. 7. Skin depth $d$ during the simulated launch for a $45^{\circ}$ copper monobloc and fibre armature.

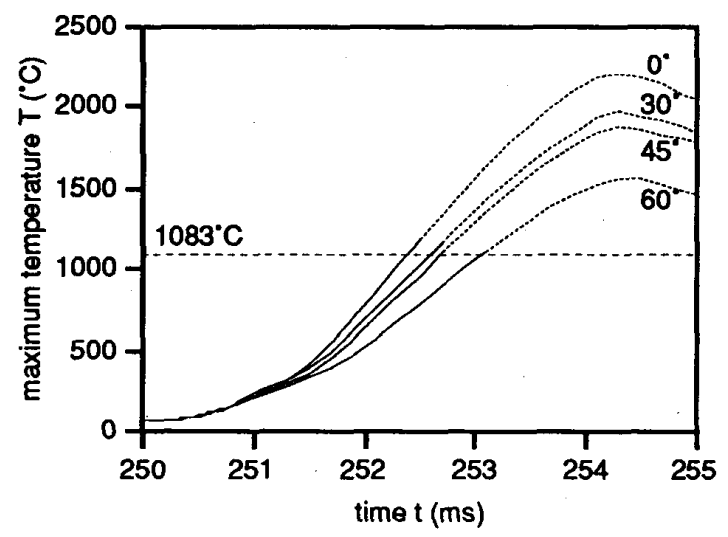

Fig. 8. Maximum temperature during the electromagnetic launch in copper fibre armatures with trailing edge angles of $0^{\circ}, 30^{\circ}, 45^{\circ}$ and $60^{\circ}$. Melting of the electrical insulation is included in the simulations, while the thermal conductivity is isotropic.

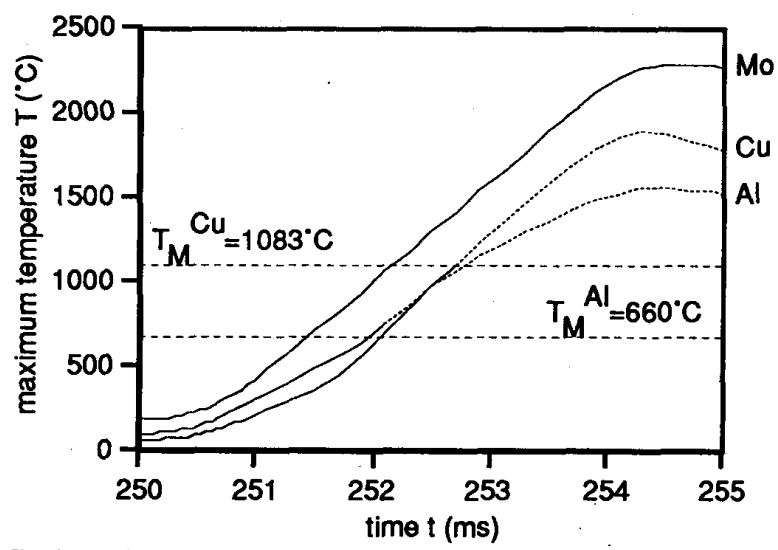

Fig. 9. Maximum temperature in $45^{\circ}$ fibre armatures of copper, aluminium and molybdenum. monobloc armatures and fibre armatures (with $r=1$ ) and copper rails with a resistive layer, were executed. The armature velocity and the breech current were taken constant and the total thickness of the rail was $17.6 \mathrm{~mm}$. The skin depth is calculated after $3 \mathrm{~ms}$, i.e. after a stationary field distribution is obtained. Several resistivities $\rho_{\mathrm{rl}}$ and thicknesses of resistive layer $w$ are considered. In these simulations, the temperature rise due to Joule heating is not taken into account. The calculated values of the skin depth for an armature velocity of $500 \mathrm{~m} / \mathrm{s}$ are given in Fig. 10 (for monobloc armatures) and Fig. 11 (for fibre armatures). For a rectangular copper monobloc armature, the optimal thicknessresistivity combination for the layer is found to be $w=1 \mathrm{~mm}$ and $\rho_{\mathrm{rl}}=100 \rho_{\mathrm{Cu}}$ for $v=500 \mathrm{~m} / \mathrm{s}$. When a smaller resistivity of the layers is chosen, the optimal layer thickness decreases, but the effect of the presence of a resistive layer on the current distribution in the armature reduces. For a lower armature velocity, thicker rail layers should be applied (see Fig. 12). These statements agree with the predictions in [6]. For rectangular copper fibre armatures, the rail layer thickness-resistivity combinations considered have only a positive effect on the current density in the armature at low velocities (e.g. $100 \mathrm{~m} / \mathrm{s}$ ). Moreover, it is unexpectedly found that a rail layer with a lower resistivity than that of the armature, will also increase the skin depth (see Fig. 11).

To study the effect of a resistive layer on the electrothermal behaviour of a $U$-shaped monobloc armature (with $\alpha=45^{\circ}$ ) during electromagnetic launch, a simulation is executed for a layer of $0.5 \mathrm{~mm}$ thickness and a resistivity 50 times that of copper (in the order of that of stainless steel). For this hypothetical layer material, values are chosen for the specific heat and the thermal conductivity equal to 0.5 and 0.1 times those of copper. The same breech current and velocity profiles are used as in section III (see Fig. 4). In Fig. 13, the maximum temperature and the skin depth in the armature during this simulated launch are compared with those during a launch where copper rails are applied. When resistive layers on the rails are used, it takes more time for the armature to

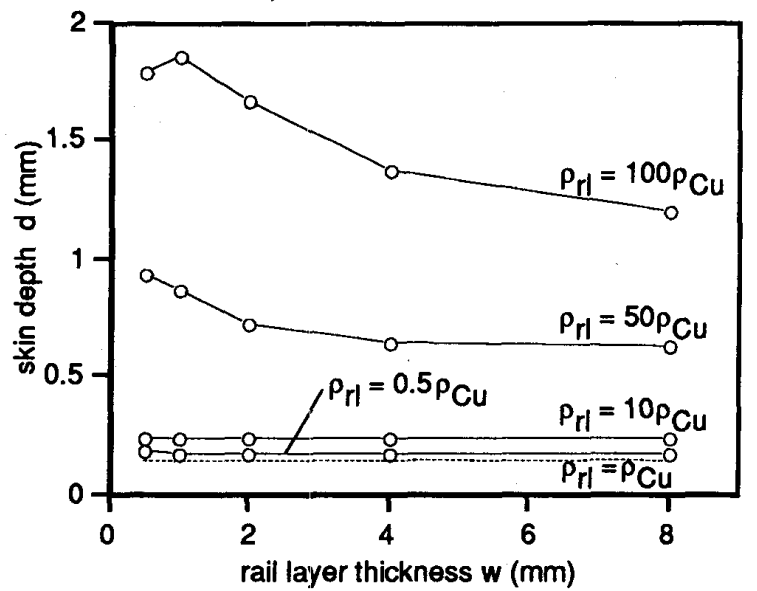

Fig. 10. Skin depth in a rectangular copper monobloc armature at $v=500$ $\mathrm{m} / \mathrm{s}$ versus the resistive rail layer thickness $w$ and resistivity $\rho_{\mathrm{rl}}$. Joule heating is neglected. 


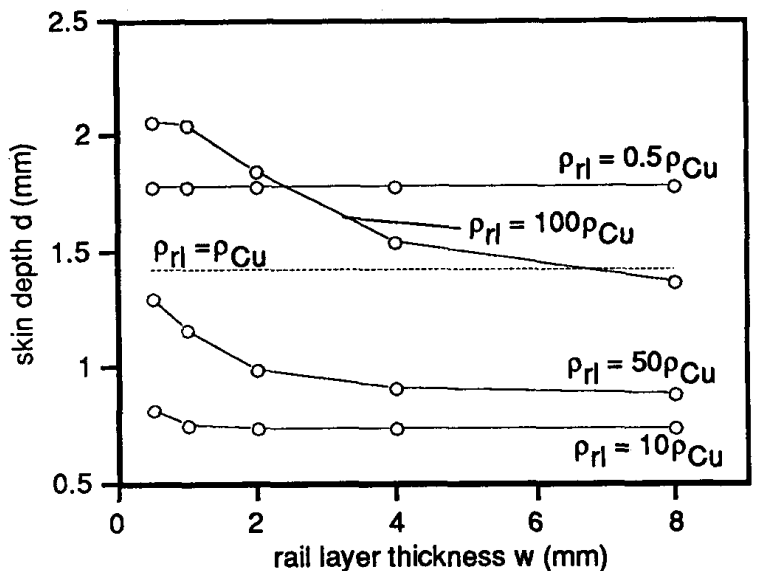

Fig. 11. Skin depth in a rectangular copper fibre armature at $y=500 \mathrm{~m} / \mathrm{s}$. Joule heating is neglected.

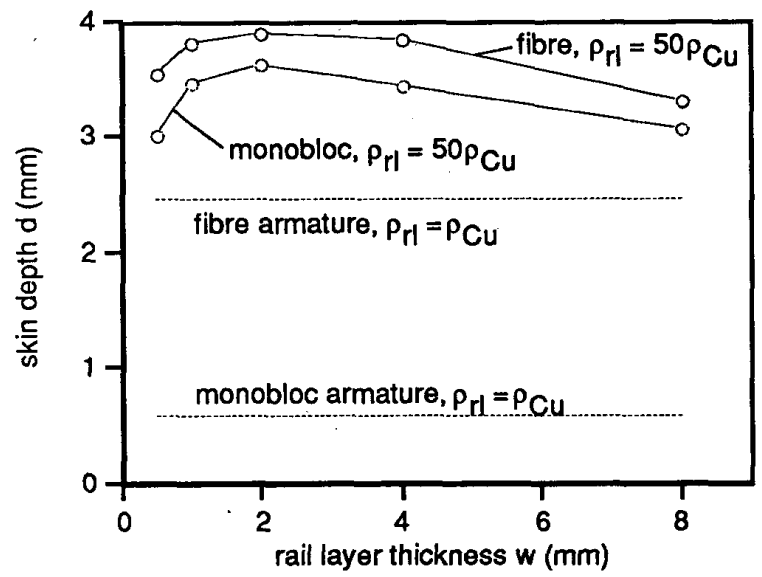

Fig. 12. Skin depth in rectangular copper monobloc and fibre armatures at $v=100 \mathrm{~m} / \mathrm{s}$. Joule heating is neglected.

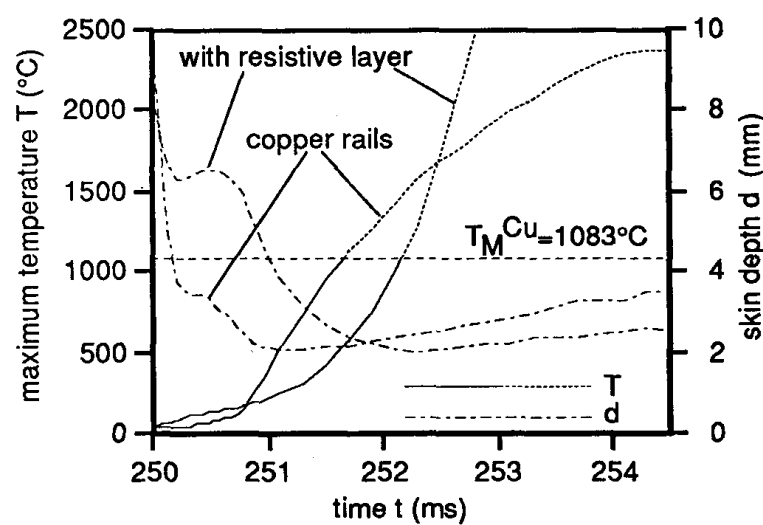

Fig. 13. Maximum temperature $T$ and skin depth $d$ in a $45^{\circ}$ copper monobloc armature during electromagnetic launch with homogeneous copper rails and with copper rails covered with a resistive layer. arrive at its melting temperature. Then, the maximum temperature in the resistive layers during the launch is $893^{\circ} \mathrm{C}$. Although the maximum temperature in the copper armature during a launch where resistive layers on the rails are used, rises very fast when the melting temperature of the armature is reached, the potential to achieve higher transition velocities for monobloc armatures under such conditions is clearly demonstrated. Unfortunately, up to now no experimental results of launching with resistively layered rails are available.

\section{CONCLUSIONS}

The effect of the application of multi-fibre solid brush armatures and resistively layered rails on the electrothermal behaviour of the armature during electromagnetic launching has been studied by means of 2-dimensional computer simulations. It is shown that the use of fibre armatures will increase the transition velocity. Especially, U-shaped molybdenum fibre armatures with a large trailing edge angle are good candidates to achieve arc erosion-free launching up to high muzzle velocities, under the condition that the electrical insulation remains unaffected during the launch process. The application of rails with a resistive layer in launches with monobloc armatures can increase the skin depth in the armature. However, the performance of a chosen rail layerarmature combination depends to a large extend on the material properties of the materials used. Therefore, more study on the application of fibre armatures in combination with resistively layered rails is necessary.

\section{ACKNOWLEDGEMENT}

The author thanks W. Karthaus, dr W.J. Kolkert and M. Koops for pleasant discussions on EML topics.

\section{REFERENCES}

[1] G.C. Long, "Fundamental limits to the velocity of solid armatures in railguns", Ph.D. thesis, The University of Texas at Austin, August 1987.

[2] G.C. Long, W.F. Weldon, "Limits to the velocity of solid armatures in railguns", IEEE Trans. Magn. 25 (1989) 347352.

[3] W.F. Hughes, F.J. Young, "Diffusion skin effects in ultrahigh velocity laminated current collectors", Wear 78 (1982) $171-178$.

[4] A.J. Schoolderman, "Two-dimensional electrothermal modelling of multi-fibre solid brush armatures", Proceedings of the 4th European Symposium on EML Technology, May 2-6, 1993, Celle, Germany.

[5] A.J. Schoolderman, W. de Zeeuw, M. Koops, "Electrothermal design aspects of transitioning solid armatures", IEEE Trans. Magn. 29 (1993) 865-870.

[6] W.F. Weldon, H.H. Woodson, G.C. Long, "Method and construction for control of current distribution in railgun armatures", U.S. patent 4,953,441, September 4, 1990.

[7] T.E. James, "Performance criteria for EM rail launchers with solid or transition armatures and laminated rails", IEEE Trans. Magn. 27 (1991) 482-487.

[8] Y.A. Dreizin, "Solid armature performance with resistive rails", IEEE Trans. Magn. 29 (1993) 798-803. 